\title{
Oscillation of second-order nonlinear dynamic equations with positive and negative coefficients
}

Da-Xue Chen ${ }^{1 *}$, Pei-Xin Qu and Yong-Hong Lan ${ }^{3}$

"Correspondence: cdx2003@163.com

${ }^{1}$ College of Science, Hunan Institute of Engineering, 88 East Fuxing Road, Xiangtan, Hunan 411104, P.R. China Full list of author information is available at the end of the article

\begin{abstract}
The paper considers the oscillation of a second-order nonlinear dynamic equation with positive and negative coefficients of the form

$$
\left[r(t) x^{\Delta}(t)\right]^{\Delta}+p(t) f(x(\xi(t)))-q(t) h(x(\delta(t)))=0
$$

on an arbitrary time scale $\mathbb{T}$. We obtain some oscillation criteria for the equation by developing a generalized Riccati substitution technique. Our results extend and improve some known results in the literature. Several examples are given to illustrate our main results.
\end{abstract}

MSC: 39A 10; 34N05

Keywords: oscillation; dynamic equation; positive and negative coefficients; time scale

\section{Introduction and preliminaries}

In this paper, we investigate the oscillation of a second-order nonlinear dynamic equation with positive and negative coefficients of the form

$$
\left[r(t) x^{\Delta}(t)\right]^{\Delta}+p(t) f(x(\xi(t)))-q(t) h(x(\delta(t)))=0
$$

on an arbitrary time scale $\mathbb{T}$ with $\sup \mathbb{T}=\infty$, subject to the following conditions:

$\left(C_{1}\right) t_{0} \in \mathbb{T}$ and $\left[t_{0}, \infty\right)_{\mathbb{T}}:=\left\{t \in \mathbb{T}: t \geq t_{0}\right\}$ is a time scale interval in $\mathbb{T}$;

$\left(\mathrm{C}_{2}\right) r \in C_{\mathrm{rd}}\left(\left[t_{0}, \infty\right)_{\mathbb{T}},(0, \infty)\right)$ and $\int_{t_{0}}^{\infty} \frac{1}{r(t)} \Delta t=\infty$;

$\left(\mathrm{C}_{3}\right) p, q \in C_{\mathrm{rd}}\left(\left[t_{0}, \infty\right)_{\mathbb{T}},[0, \infty)\right)$;

$\left(C_{4}\right) \xi, \delta \in C_{\mathrm{rd}}(\mathbb{T}, \mathbb{T}), \lim _{t \rightarrow \infty} \xi(t)=\lim _{t \rightarrow \infty} \delta(t)=\infty, \delta$ has the inverse function $\delta^{-1} \in$ $C_{\mathrm{rd}}(\mathbb{T}, \mathbb{T}), v:=\delta^{-1} \circ \xi \in C_{\mathrm{rd}}(\mathbb{T}, \mathbb{T}), \xi^{\Delta}, v^{\Delta} \in C_{\mathrm{rd}}\left(\left[t_{0}, \infty\right)_{\mathbb{T}},(0, \infty)\right), \xi(t), v(t) \leq t$ for $t \in$ $\left[t_{0}, \infty\right)_{\mathbb{T}}, \quad \xi\left(\left[t_{0}, \infty\right)_{\mathbb{T}}\right)=\left[\xi\left(t_{0}\right), \infty\right)_{\mathbb{T}}$ and $v\left(\left[t_{0}, \infty\right)_{\mathbb{T}}\right)=\left[v\left(t_{0}\right), \infty\right)_{\mathbb{T}}$, where $\xi\left(\left[t_{0}, \infty\right)_{\mathbb{T}}\right):=\left\{\xi(t): t \in\left[t_{0}, \infty\right)_{\mathbb{T}}\right\}$ and $v\left(\left[t_{0}, \infty\right)_{\mathbb{T}}\right):=\left\{v(t): t \in\left[t_{0}, \infty\right)_{\mathbb{T}}\right\} ;$

$\left(\mathrm{C}_{5}\right) f, h \in C(\mathbb{R}, \mathbb{R})$, there exist positive constants $L_{1}, L_{2}$ and $M$ such that $f(u) / u \geq L_{1}, 0<$ $h(u) / u \leq L_{2}$ and $|h(u)| \leq M$ for $u \neq 0$, and $L_{1} p(t)-L_{2} q(v(t)) v^{\Delta}(t)>0$ for $t \in\left[t_{0}, \infty\right)_{\mathbb{T}} ;$ $\left(\mathrm{C}_{6}\right) \int_{t}^{\infty}\left[\frac{1}{r(s)} \int_{v(s)}^{s} q(u) \Delta u\right] \Delta s<\infty$ for every sufficiently large $t \in \mathbb{T}$.

Recall that a solution of $(1)$ is a nontrivial real function $x$ such that $x \in C_{\mathrm{rd}}^{1}\left(\left[t_{x}, \infty\right)_{\mathbb{T}}, \mathbb{R}\right)$ and $r x^{\Delta} \in C_{\mathrm{rd}}^{1}\left(\left[t_{x}, \infty\right)_{\mathbb{T}}, \mathbb{R}\right)$ for a certain $t_{x} \geq t_{0}$ and satisfying (1) for $t \geq t_{x}$. Our attention is 
restricted to those solutions of (1) which exist on $\left[t_{x}, \infty\right)_{\mathbb{T}}$ and satisfy $\sup \left\{|x(t)|: t>t_{*}\right\}>0$ for any $t_{*} \geq t_{x}$. A solution $x$ of (1) is said to be oscillatory if it is neither eventually positive nor eventually negative; otherwise, it is nonoscillatory. Equation (1) is said to be oscillatory if all its solutions are oscillatory.

For convenience of the readers and completeness of the paper, we recall the following basic concepts and results for the calculus on time scales. More details can be found in [1, 2].

A time scale $\mathbb{T}$ is an arbitrary nonempty closed subset of the real numbers $\mathbb{R}$. We assume throughout that $\mathbb{T}$ has the topology that it inherits from the standard topology on the real numbers $\mathbb{R}$. Some examples of time scales are as follows: the real numbers $\mathbb{R}$, the integers $\mathbb{Z}$, the positive integers $\mathbb{N}$, the nonnegative integers $\mathbb{N}_{0},[0,1] \cup[2,3],[0,1] \cup \mathbb{N}$, $h \mathbb{Z}:=\{h k: k \in \mathbb{Z}, h>0\}$ and $\overline{q^{\mathbb{Z}}}:=\left\{q^{k}: k \in \mathbb{Z}, q>1\right\} \cup\{0\}$. But the rational numbers $\mathbb{Q}$, the complex numbers $\mathbb{C}$ and the open interval $(0,1)$ are not time scales. Many other interesting time scales exist, and they give rise to plenty of applications (see [1]).

For $t \in \mathbb{T}$, the forward jump operator and the backward jump operator are defined by

$$
\sigma(t):=\inf \{s \in \mathbb{T}: s>t\} \quad \text { and } \quad \rho(t):=\sup \{s \in \mathbb{T}: s<t\}
$$

respectively, where we put $\inf \emptyset=\sup \mathbb{T}$ (i.e., $\sigma(t)=t$ if $\mathbb{T}$ has a maximum $t$ ) and $\sup \emptyset=$ $\inf \mathbb{T}$ (i.e., $\rho(t)=t$ if $\mathbb{T}$ has a minimum $t$ ), here $\emptyset$ denotes the empty set. It is easy to see

$$
\rho(t) \leq t \leq \sigma(t) \quad \text { for } t \in \mathbb{T} \text {. }
$$

Let $t \in \mathbb{T}$. If $\sigma(t)>t$, we say that $t$ is right-scattered, while if $\rho(t)<t$, we say that $t$ is left-scattered. Points that are right-scattered and left-scattered at the same time are called isolated. Also, if $t<\sup \mathbb{T}$ and $\sigma(t)=t$, then $t$ is called right-dense, and if $t>\inf \mathbb{T}$ and $\rho(t)=t$, then $t$ is called left-dense. The graininess function $\mu: \mathbb{T} \rightarrow[0, \infty)$ is defined by

$$
\mu(t):=\sigma(t)-t
$$

We also need below the set $\mathbb{T}^{\kappa}$ : If $\mathbb{T}$ has a left-scattered maximum $m$, then $\mathbb{T}^{\kappa}=\mathbb{T}-\{m\}$. Otherwise, $\mathbb{T}^{\kappa}=\mathbb{T}$. Let $f: \mathbb{T} \rightarrow \mathbb{R}$, then we define the function $f^{\sigma}: \mathbb{T}^{\kappa} \rightarrow \mathbb{R}$ by

$$
f^{\sigma}(t):=f(\sigma(t)) \quad \text { for all } t \in \mathbb{T}^{\kappa},
$$

i.e., $f^{\sigma}:=f \circ \sigma$.

For $a, b \in \mathbb{T}$ with $a<b$, we define the time scale interval $[a, b]_{\mathbb{T}}$ in $\mathbb{T}$ by

$$
[a, b]_{\mathbb{T}}:=\{t \in \mathbb{T}: a \leq t \leq b\}
$$

Open time scale intervals and half-open time scale intervals etc. are defined accordingly.

Fix $t \in \mathbb{T}^{\kappa}$ and let $f: \mathbb{T} \rightarrow \mathbb{R}$. Define $f^{\Delta}(t)$ to be the number (provided it exists) with the property that given any $\varepsilon>0$, there is a neighborhood $U$ of $t$ such that

$$
\left|[f(\sigma(t))-f(s)]-f^{\Delta}(t)[\sigma(t)-s]\right| \leq \varepsilon|\sigma(t)-s| \quad \text { for all } s \in U \text {. }
$$


In this case, we say that $f^{\Delta}(t)$ is the (delta) derivative of $f$ at $t$ and that $f$ is (delta) differentiable at $t$.

If the time scale $\mathbb{T}$ is the real numbers $\mathbb{R}$, then the usual derivative is retrieved, that is,

$$
f^{\Delta}(t)=f^{\prime}(t) \quad \text { for } t \in \mathbb{R}
$$

If the time scale $\mathbb{T}$ is taken to be the integers $\mathbb{Z}$, then the delta derivative reduces to the usual forward difference, that is,

$$
f^{\Delta}(t)=\Delta f(t)=f(t+1)-f(t) \quad \text { for } t \in \mathbb{Z} .
$$

Assume that $f: \mathbb{T} \rightarrow \mathbb{R}$ and let $t \in \mathbb{T}^{\kappa}$. If $f$ is (delta) differentiable at $t$, then

$$
f(\sigma(t))=f(t)+\mu(t) f^{\Delta}(t)
$$

If $\mu(t) \neq 0$, then from (4) we have

$$
f^{\Delta}(t)=\frac{f(\sigma(t))-f(t)}{\mu(t)} .
$$

A function $f: \mathbb{T} \rightarrow \mathbb{R}$ is said to be right-dense continuous (rd-continuous) provided it is continuous at each right-dense point in $\mathbb{T}$ and its left-sided limits exist (finite) at all left-dense points in $\mathbb{T}$. The set of all such rd-continuous functions is denoted by

$$
C_{\mathrm{rd}}(\mathbb{T})=C_{\mathrm{rd}}(\mathbb{T}, \mathbb{R})
$$

The set of functions $f: \mathbb{T} \rightarrow \mathbb{R}$ that are (delta) differentiable and whose (delta) derivative is rd-continuous is denoted by

$$
C_{\mathrm{rd}}^{1}(\mathbb{T})=C_{\mathrm{rd}}^{1}(\mathbb{T}, \mathbb{R})
$$

We will make use of the following product and quotient rules for the (delta) derivatives of the product $f g$ and the quotient $f / g$ of two (delta) differentiable functions $f$ and $g$ :

$$
(f g)^{\Delta}=f^{\Delta} g+f^{\sigma} g^{\Delta}=f g^{\Delta}+f^{\Delta} g^{\sigma}
$$

and

$$
\left(\frac{f}{g}\right)^{\Delta}=\frac{f^{\Delta} g-f g^{\Delta}}{g g^{\sigma}}=\frac{f^{\Delta}}{g^{\sigma}}-\frac{g^{\Delta} f}{g g^{\sigma}},
$$

where $g^{\sigma}=g \circ \sigma$ and $g g^{\sigma} \neq 0$.

For $a, b \in \mathbb{T}$ and a (delta) differentiable function $f$, the Cauchy (delta) integral of $f^{\Delta}$ is defined by

$$
\int_{a}^{b} f^{\Delta}(t) \Delta t=f(b)-f(a) .
$$


The integration by parts formula reads

$$
\int_{a}^{b} f(t) g^{\Delta}(t) \Delta t=f(b) g(b)-f(a) g(a)-\int_{a}^{b} f^{\Delta}(t) g^{\sigma}(t) \Delta t
$$

or

$$
\int_{a}^{b} f^{\sigma}(t) g^{\Delta}(t) \Delta t=f(b) g(b)-f(a) g(a)-\int_{a}^{b} f^{\Delta}(t) g(t) \Delta t .
$$

The infinite integral is defined as

$$
\int_{a}^{\infty} f(s) \Delta s=\lim _{t \rightarrow \infty} \int_{a}^{t} f(s) \Delta s
$$

The calculus on time scales was introduced by Hilger [3] with the motivation of providing a unified approach to continuous and discrete calculus. The theory of dynamic equations on time scales not only unifies the theories of differential equations and difference equations, but it is also able to extend these classical cases to cases 'in between', e.g., to the so-called $q$-difference equations. Dynamic equations on time scales have an enormous potential for modeling a variety of applications; see, for example, the monograph by Bohner and Peterson [1]. For advances in dynamic equations on time scales, one can see the book by Bohner and Peterson [2].

In recent years, there has been much research activity concerning the oscillation, nonoscillation and asymptotic behavior of solutions of various dynamic equations and differential equations. For instance, Došlý and Hilger [4] considered the second-order Sturm-Liouville dynamic equation

$$
\left[r(t) x^{\Delta}(t)\right]^{\Delta}+p(t) x^{\sigma}(t)=0 \quad \text { for } t \in \mathbb{T},
$$

where $\sigma$ is the forward jump operator on $\mathbb{T}, x^{\sigma}:=x \circ \sigma, r(t) \neq 0, r$ and $p$ are rd-continuous functions and $\lim _{s \rightarrow t^{-}} r(s) \neq 0$ at all left-dense and right-scattered points. They established a necessary and sufficient condition for the oscillation of (10) by using the so-called trigonometric transformation.

Medico and Kong [5, 6] also investigated the oscillation of (10). They supposed that $r, p \in C_{\mathrm{rd}}(\mathbb{T}, \mathbb{R})$ with $r(t)>0$. Medico and Kong [5] gave some Kamenev-type and interval criteria for the oscillation of (10). Their results covered those for differential equations and offered new oscillation criteria for difference equations. Medico and Kong [6] extended the work in [5] by modifying the class of kernel functions and deriving new criteria of Sun type (see [7]).

Saker [8] obtained some oscillation criteria for the second-order nonlinear dynamic equation

$$
\left[r(t) x^{\Delta}(t)\right]^{\Delta}+p(t) f\left(x^{\sigma}(t)\right)=0
$$

on time scales in terms of the coefficients and the graininess function, where $r$ and $p$ are positive real-valued rd-continuous functions, $\int_{t_{0}}^{\infty} \frac{1}{r(t)} \Delta t=\infty$ or $\int_{t_{0}}^{\infty} \frac{1}{r(t)} \Delta t<\infty$, and $f$ : $\mathbb{R} \rightarrow \mathbb{R}$ such that $x f(x)>0$ and $f(x) / x \geq K>0$ for $x \neq 0$. 
Erbe and Peterson [9] also discussed the oscillation of (11), where $r(t)>0$. When no explicit sign assumptions are made with respect to the coefficient $p$, they established some sufficient conditions for the oscillation of (11) when $\liminf _{t \rightarrow \infty} \int_{T}^{t} p(t) \Delta t>0$ for sufficiently large $T$.

Zhang and Zhu [10] studied the oscillation of the second-order nonlinear dynamic equations

$$
x^{\Delta \Delta}(t)+p(t) f\left(x\left(t-\xi_{0}\right)\right)=0
$$

and

$$
x^{\Delta \Delta}(t)+p(t) f\left(x^{\sigma}(t)\right)=0
$$

on a time scale $\mathbb{T}$, where $\xi_{0} \in \mathbb{R}, t-\xi_{0} \in \mathbb{T}, p: \mathbb{T} \rightarrow[0, \infty)$ is a real-valued rd-continuous function, $f: \mathbb{R} \rightarrow \mathbb{R}$ is continuous, $f(u)$ is nondecreasing, $f(-u)=-f(u)$ for $u \in \mathbb{R}$, and $u f(u)>0$ for $u \neq 0$. They established the equivalence of the oscillation of (12) and (13), from which they obtained some oscillation criteria and a comparison theorem for (12).

Şahiner [11] got some sufficient conditions for the oscillation of the second-order nonlinear delay dynamic equation

$$
x^{\Delta \Delta}(t)+p(t) f(x(\xi(t)))=0
$$

on a time scale interval $\left[t_{0}, \infty\right)_{\mathbb{T}}$, where $p \in C_{\mathrm{rd}}(\mathbb{T}, \mathbb{R})$ is a positive function, $\xi \in C_{\mathrm{rd}}(\mathbb{T}, \mathbb{T})$ is an increasing function such that $\xi(t)<t$ and $\lim _{t \rightarrow \infty} \xi(t)=\infty$, and $f \in C(\mathbb{R}, \mathbb{R})$ satisfies $f(x) / x \geq L$ for a certain positive constant $L$ and for all $x \neq 0$.

Erbe et al. [12] were concerned with the oscillation of the second-order nonlinear delay dynamic equation

$$
\left[r(t) x^{\Delta}(t)\right]^{\Delta}+p(t) f(x(\xi(t)))=0 \quad \text { for } t \in\left[t_{0}, \infty\right)_{\mathbb{T}},
$$

where $r$ and $p$ are real rd-continuous positive functions defined on $\mathbb{T}$, the so-called delay function $\xi$ satisfies $\xi:\left[t_{0}, \infty\right)_{\mathbb{T}} \rightarrow \mathbb{T}$ is rd-continuous, $\xi(t) \leq t$ for $t \in\left[t_{0}, \infty\right)_{\mathbb{T}}$, $\lim _{t \rightarrow \infty} \xi(t)=\infty$, and $f: \mathbb{R} \rightarrow \mathbb{R}$ is a continuous function satisfying $u f(u)>0$ for all $u \neq 0$ and $|f(u)| \geq K|u|$. The authors obtained some new oscillation criteria which improved the results established by Zhang and Zhu [10] and Şahiner [11].

Jia et al. [13] also dealt with the oscillation of (13), where $p \in C_{\mathrm{rd}}(\mathbb{T}, \mathbb{R}), \mathbb{T}$ is a time scale, and $f: \mathbb{R} \rightarrow \mathbb{R}$ is continuously differentiable and satisfies $f^{\prime}(x)>0$ and $x f(x)>0$ for $x \neq 0$. Jia et al. [13] obtained several Kiguradze-type oscillation theorems for (13).

Karpuz and Öcalan [14] studied the asymptotic behavior of a delay dynamic equation having the following form:

$$
[x(t)+A(t) x(\alpha(t))]^{\Delta}+B(t) F(x(\beta(t)))-C(t) G(x(\gamma(t)))=\varphi(t) \quad \text { for } t \in\left[t_{0}, \infty\right)_{\mathbb{T}},
$$

where $\mathbb{T}$ is a time scale unbounded from above, $F, G \in C_{\mathrm{rd}}(\mathbb{R}, \mathbb{R}), A, \varphi \in C_{\mathrm{rd}}\left(\left[t_{0}, \infty\right)_{\mathbb{T}}, \mathbb{R}\right)$, $B, C \in C_{\mathrm{rd}}\left(\left[t_{0}, \infty\right)_{\mathbb{T}},[0, \infty)_{\mathbb{R}}\right)$, and $\alpha, \beta$ and $\gamma$ are delay functions. The authors also ex- 
tended their results to the equation of the form

$$
[x(t)+A(t) x(\alpha(t))]^{\Delta}+B(t) F(x(\beta(t)))=\varphi(t) \quad \text { for } t \in\left[t_{0}, \infty\right)_{\mathbb{T}},
$$

where $B \in C_{\mathrm{rd}}\left(\left[t_{0}, \infty\right)_{\mathbb{T}}, \mathbb{R}\right)$ is allowed to oscillate.

In [15], Karpuz et al. discussed the neutral delay dynamic equation

$$
[x(t)+A(t) x(\alpha(t))]^{\Delta}+B(t) F(x(\beta(t)))-C(t) F(x(\gamma(t)))=\varphi(t) \quad \text { for } t \in\left[t_{0}, \infty\right)_{\mathbb{T}},
$$

where $\sup \mathbb{T}=\infty, A \in C_{\mathrm{rd}}\left(\left[t_{0}, \infty\right)_{\mathbb{T}}, \mathbb{R}\right), B, C \in C_{\mathrm{rd}}\left(\left[t_{0}, \infty\right)_{\mathbb{T}},(0, \infty)_{\mathbb{R}}\right), F \in C_{\mathrm{rd}}(\mathbb{R}, \mathbb{R})$, and $\alpha, \beta, \gamma \in C_{\mathrm{rd}}\left(\left[t_{0}, \infty\right)_{\mathbb{T}}, \mathbb{T}\right)$ are strictly increasing and unbounded functions. The authors weakened the assumptions on the coefficients that are assumed to hold in the literature and improved some known results by providing necessary and sufficient conditions for the solutions of the equation to oscillate or to converge to zero. The coefficient associated with the neutral part was considered in three distinct ranges, in one of which the coefficient is allowed to oscillate.

Karpuz et al. [16] obtained some necessary and sufficient conditions for every solution of the higher-order neutral functional differential equation

$$
(y(t)-p(t) y(r(t)))^{(n)}+q(t) G(y(g(t)))-u(t) H(y(h(t)))=f(t)
$$

to oscillate or to tend to zero as $t$ tends to infinity, where $n \geq 2$ is an integer, $q>0$ and $u \geq 0$. Both bounded and unbounded solutions were considered in this paper.

For some recent other results on the oscillation, nonoscillation and asymptotic behavior of solutions of different types of dynamic equations, we refer the reader to the papers [17$44]$ and the references cited therein.

The results in $[4-6,8-16]$ are very valuable. But these results also have some disadvantages. For example, the oscillation criteria of Došlý and Hilger [4] are unsatisfactory since additional assumptions have to be imposed on the unknown solutions.

The results of Zhang and Zhu [10] are valid only when the graininess function $\mu(t)$ is bounded, which is a restrictive condition (e.g., the results cannot be applied to $\mathbb{T}=q^{\mathbb{N} 0}:=$ $\left\{q^{k}: k \in \mathbb{N}_{0}, q>1\right\}$, where $\mu(t)=(q-1) t$ is unbounded; see [12]).

For (14), Şahiner proved that if there exists a delta differentiable function $\varphi$ such that for some positive constant $k \in(0,1)$,

$$
\limsup _{t \rightarrow \infty} \int_{t_{0}}^{t}\left[p(s) \varphi^{\sigma}(s) \frac{\xi(s)}{\sigma(s)}-\frac{\sigma(s)\left(\varphi^{\Delta}(s)\right)^{2}}{4 L k^{2} \xi(s) \varphi^{\sigma}(s)}\right] \Delta s=\infty
$$

then every solution of (14) oscillates. We observe that the condition (16) depends on an additional constant $k \in(0,1)$, which implies that the results are not sharp. As a special case (take $\varphi(s)=s)$, he deduced that if

$$
\limsup _{t \rightarrow \infty} \int_{t_{0}}^{t}\left[p(s) \xi(s)-\frac{1}{4 L k^{2} \xi(s)}\right] \Delta s=\infty
$$


then every solution of (14) oscillates. However, Erbe et al. [12] showed that the dynamic equation

$$
x^{\Delta \Delta}(t)+\frac{\beta}{t \xi(t)} x(\xi(t))=0
$$

is oscillatory if $\beta>\frac{1}{4}$, but (17) does not give this result.

The restriction $f^{\prime}(x)>0$ for $x \neq 0$ is required in [13]. This condition does not hold and cannot be applied in the case when $f(x)=x\left(\frac{1}{9}+\frac{1}{1+x^{2}}\right)$, since $f^{\prime}(x)=\frac{\left(x^{2}-2\right)\left(x^{2}-5\right)}{9\left(1+x^{2}\right)^{2}}$ changes sign four times.

The results in $[14,15]$ cannot be applied to higher-order delay dynamic equations with positive and negative coefficients. The results in [16] fail to be applied to general time scales.

Besides the above-mentioned disadvantages, it is clear that (10)-(15) are some special cases of (1), and that the results in [4-6, 8-13] cannot be applied to general cases of (1). Therefore, it is of great interest to investigate the oscillation of (1). To the best of our knowledge, nothing is known regarding the oscillatory behavior of (1) on time scales up to now. Following the trend shown in [4-6, 8-16], in this paper we deal with the oscillation of (1). We obtain some oscillation criteria for (1) by developing a generalized Riccati substitution technique. Our results are essentially new and extend and improve some results in [4-6, $8-13]$. We also illustrate our main results with several examples.

In what follows, for convenience, when we write a functional inequality or equality without specifying its domain of validity, we assume that it holds for all sufficiently large $t$.

\section{Lemmas}

Lemma 2.1 (Substitution [1, Theorem 1.98]) Assume that $\eta: \mathbb{T} \rightarrow \mathbb{R}$ is strictly increasing and that $\widetilde{\mathbb{T}}:=\eta(\mathbb{T}):=\{\eta(t): t \in \mathbb{T}\}$ is a time scale. If $: \mathbb{T} \rightarrow \mathbb{R}$ is an $r d$-continuous function, $\eta$ is differentiable with $r d$-continuous derivative, and $a, b \in \mathbb{T}$, then

$$
\int_{a}^{b} f(t) \eta^{\Delta}(t) \Delta t=\int_{\eta(a)}^{\eta(b)}\left(f \circ \eta^{-1}\right)(s) \widetilde{\Delta} s
$$

where $\eta^{-1}$ is the inverse function of $\eta$ and $\widetilde{\Delta}$ denotes the derivative on $\widetilde{\mathbb{T}}$.

Lemma 2.2 (Existence of antiderivatives [1, Theorem 1.74]) Every rd-continuous function has an antiderivative. In particular if $t_{0} \in \mathbb{T}$, then $F$ defined by

$$
F(t):=\int_{t_{0}}^{t} f(\tau) \Delta \tau \quad \text { for } t \in \mathbb{T}
$$

is an antiderivative of $f$.

Lemma 2.3 (Chain rule [1, Theorem 1.93]) Assume that $\vartheta: \mathbb{T} \rightarrow \mathbb{R}$ is strictly increasing and that $\widetilde{\mathbb{T}}:=\vartheta(\mathbb{T}):=\{\vartheta(t): t \in \mathbb{T}\}$ is a time scale. Let $\varphi: \widetilde{\mathbb{T}} \rightarrow \mathbb{R}$. If $\vartheta^{\Delta}(t)$ and $\varphi^{\widetilde{\Delta}}(\vartheta(t))$ exist for $t \in \mathbb{T}^{\kappa}$, then

$$
(\varphi \circ \vartheta)^{\Delta}=\left(\varphi^{\widetilde{\Delta}} \circ \vartheta\right) \vartheta^{\Delta},
$$

where ${ }^{\widetilde{\Delta}}$ denotes the derivative on $\widetilde{\mathbb{T}}$. 


\section{Main results}

Theorem 3.1 Assume that $\left(\mathrm{C}_{1}\right)-\left(\mathrm{C}_{6}\right)$ hold. Furthermore, suppose that there exists a positive function $a \in C_{\mathrm{rd}}^{1}\left(\left[t_{0}, \infty\right)_{\mathbb{T}}, \mathbb{R}\right)$ such that for every sufficiently large $T$,

$$
\limsup _{t \rightarrow \infty} \int_{T}^{t}\left\{a(s)\left[L_{1} p(s)-L_{2} q(v(s)) v^{\Delta}(s)\right]-\frac{\left(a_{+}^{\Delta}(s)\right)^{2} r(\xi(s))}{4 a(s) \xi^{\Delta}(s)}\right\} \Delta s=\infty
$$

where $a_{+}^{\Delta}(s):=\max \left\{a^{\Delta}(s), 0\right\}$. Then every solution of $(1)$ is oscillatory.

Proof Suppose that $x$ is a nonoscillatory solution of (1). Without loss of generality, we may assume that $x$ is an eventually positive solution of (1). For every sufficiently large $t \in \mathbb{T}$, define the function $z$ by

$$
z(t):=x(t)+\int_{t}^{\infty}\left[\frac{1}{r(s)} \int_{v(s)}^{s} q(u) h(x(\delta(u))) \Delta u\right] \Delta s
$$

where $v$ is defined as in $\left(\mathrm{C}_{4}\right)$. From $\left(\mathrm{C}_{6}\right)$ and the boundedness of $h$, we see that $z(t)$ is well defined for every sufficiently large $t \in \mathbb{T}$. It follows from (19) that

$$
z(t) \geq x(t)>0
$$

and

$$
z^{\Delta}(t)=x^{\Delta}(t)-\frac{1}{r(t)} \int_{v(t)}^{t} q(u) h(x(\delta(u))) \Delta u .
$$

Therefore, there exists a sufficiently large $t_{1} \in\left[t_{0}, \infty\right)_{\mathbb{T}}$ such that for $t \in\left[t_{1}, \infty\right)_{\mathbb{T}}$,

$$
\begin{aligned}
r(t) z^{\Delta}(t) & =r(t) x^{\Delta}(t)-\int_{v(t)}^{t} q(u) h(x(\delta(u))) \Delta u \\
& =r(t) x^{\Delta}(t)-\int_{v\left(t_{1}\right)}^{t} q(u) h(x(\delta(u))) \Delta u+\int_{v\left(t_{1}\right)}^{v(t)} q(u) h(x(\delta(u))) \Delta u .
\end{aligned}
$$

Making the substitution $s=v(u)$, from Lemma 2.1 and $\left(\mathrm{C}_{4}\right)$, we have

$$
\begin{aligned}
\int_{t_{1}}^{t} q(v(u)) h(x(\xi(u))) v^{\Delta}(u) \Delta u & =\int_{v\left(t_{1}\right)}^{v(t)} q(s) h\left(x\left(\xi\left(v^{-1}(s)\right)\right)\right) \widetilde{\Delta} s \\
& =\int_{v\left(t_{1}\right)}^{v(t)} q(s) h(x(\delta(s))) \widetilde{\Delta} s \quad \text { for } t \in\left[t_{1}, \infty\right)_{\mathbb{T}},
\end{aligned}
$$

where $v^{-1}$ is the inverse function of $v$. According to the condition $v\left(\left[t_{0}, \infty\right)_{\mathbb{T}}\right)=\left[v\left(t_{0}\right), \infty\right)_{\mathbb{T}}$ in $\left(C_{4}\right)$, we get that the derivative ${ }^{\Delta}$ on $\mathbb{T}$ is equal to the derivative ${ }^{\widetilde{\Delta}}$ on $\widetilde{T}:=v\left(\left[t_{0}, \infty\right)_{\mathbb{T}}\right)$ in (23). Hence, from (23) we conclude

$$
\int_{t_{1}}^{t} q(v(u)) h(x(\xi(u))) v^{\Delta}(u) \Delta u=\int_{v\left(t_{1}\right)}^{v(t)} q(s) h(x(\delta(s))) \Delta s \quad \text { for } t \in\left[t_{1}, \infty\right)_{\mathbb{T}} .
$$


From (22) and (24), it follows that for $t \in\left[t_{1}, \infty\right)_{\mathbb{T}}$,

$$
\begin{aligned}
r(t) z^{\Delta}(t)= & r(t) x^{\Delta}(t)-\int_{v\left(t_{1}\right)}^{t} q(u) h(x(\delta(u))) \Delta u \\
& +\int_{t_{1}}^{t} q(v(u)) h(x(\xi(u))) v^{\Delta}(u) \Delta u .
\end{aligned}
$$

By Lemma 2.2 and (25), we obtain for $t \in\left[t_{1}, \infty\right)_{\mathbb{T}}$,

$$
\left[r(t) z^{\Delta}(t)\right]^{\Delta}=\left[r(t) x^{\Delta}(t)\right]^{\Delta}-q(t) h(x(\delta(t)))+q(v(t)) h(x(\xi(t))) v^{\Delta}(t) .
$$

From (1) and (26), it follows that

$$
\left[r(t) z^{\Delta}(t)\right]^{\Delta}=-p(t) f(x(\xi(t)))+q(v(t)) h(x(\xi(t))) v^{\Delta}(t) .
$$

Hence, from $\left(\mathrm{C}_{4}\right),\left(\mathrm{C}_{5}\right)$ and (27), we conclude

$$
\begin{aligned}
{\left[r(t) z^{\Delta}(t)\right]^{\Delta} } & \leq-p(t) L_{1} x(\xi(t))+q(v(t)) L_{2} x(\xi(t)) v^{\Delta}(t) \\
& :=-Q(t) x(\xi(t)) \\
& <0,
\end{aligned}
$$

where $Q(t):=L_{1} p(t)-L_{2} q(v(t)) v^{\Delta}(t)$. Thus, there exists $t_{2} \in\left[t_{1}, \infty\right)_{\mathbb{T}}$ such that $r(t) z^{\Delta}(t)$ is strictly decreasing on $\left[t_{2}, \infty\right)_{\mathbb{T}}$ and is either eventually positive or eventually negative. Since $r(t)>0$ for $t \in\left[t_{0}, \infty\right)_{\mathbb{T}}, z^{\Delta}(t)$ is also either eventually positive or eventually negative. We claim

$$
z^{\Delta}(t)>0 \text { for } t \in\left[t_{2}, \infty\right)_{\mathbb{T}} .
$$

Assume that (29) does not hold, then there exists $t_{3} \in\left[t_{2}, \infty\right)_{\mathbb{T}}$ such that $z^{\Delta}\left(t_{3}\right)<0$. Since $r(t) z^{\Delta}(t)$ is strictly decreasing on $\left[t_{2}, \infty\right)_{\mathbb{T}}$, it is clear that $r(t) z^{\Delta}(t) \leq r\left(t_{3}\right) z^{\Delta}\left(t_{3}\right):=-c<0$ for $t \in\left[t_{3}, \infty\right)_{\mathbb{T}}$. Thus, we obtain $z^{\Delta}(t) \leq-c \frac{1}{r(t)}$ for $t \in\left[t_{3}, \infty\right)_{\mathbb{T}}$. By integrating both sides of the last inequality from $t_{3}$ to $t$, we get

$$
z(t)-z\left(t_{3}\right) \leq-c \int_{t_{3}}^{t} \frac{1}{r(s)} \Delta s \quad \text { for } t \in\left[t_{3}, \infty\right)_{\mathbb{T}}
$$

Noticing $\left(\mathrm{C}_{2}\right)$ and letting $t \rightarrow \infty$, we see $\lim _{t \rightarrow \infty} z(t)=-\infty$. This contradicts $(20)$. Therefore, (29) holds. From (21) and (29), we have

$$
x^{\Delta}(t) \geq z^{\Delta}(t)>0 \text {. }
$$

Define the function $w$ by the generalized Riccati substitution

$$
w(t):=a(t) \frac{r(t) z^{\Delta}(t)}{x(\xi(t))} .
$$


It is easy to see that there exists $t_{4} \in\left[t_{3}, \infty\right)_{\mathbb{T}}$ such that $w(t)>0$ for $t \in\left[t_{4}, \infty\right)_{\mathbb{T}}$. Using (6) and (7), from (31) we get

$$
\begin{aligned}
w^{\Delta} & =\left(r z^{\Delta}\right)^{\Delta} \frac{a}{x \circ \xi}+\left(r z^{\Delta}\right)^{\sigma}\left(\frac{a}{x \circ \xi}\right)^{\Delta} \\
& =\left(r z^{\Delta}\right)^{\Delta} \frac{a}{x \circ \xi}+\left(r z^{\Delta}\right)^{\sigma}\left[\frac{a^{\Delta}}{(x \circ \xi)^{\sigma}}-\frac{(x \circ \xi)^{\Delta} a}{(x \circ \xi)(x \circ \xi)^{\sigma}}\right] \\
& \leq\left(r z^{\Delta}\right)^{\Delta} \frac{a}{x \circ \xi}+a_{+}^{\Delta} \frac{w^{\sigma}}{a^{\sigma}}-a \frac{w^{\sigma}}{a^{\sigma}} \frac{(x \circ \xi)^{\Delta}}{x \circ \xi},
\end{aligned}
$$

where $a_{+}^{\Delta}$ is defined as in Theorem 3.1. From (28) and (32) we have

$$
w^{\Delta} \leq-Q a+a_{+}^{\Delta} \frac{w^{\sigma}}{a^{\sigma}}-a \frac{w^{\sigma}}{a^{\sigma}} \frac{(x \circ \xi)^{\Delta}}{x \circ \xi} .
$$

From $\left(\mathrm{C}_{4}\right)$ and Lemma 2.3, we find

$$
(x \circ \xi)^{\Delta}=\left(x^{\widetilde{\Delta}} \circ \xi\right) \xi^{\Delta} .
$$

According to the condition $\xi\left(\left[t_{0}, \infty\right)_{\mathbb{T}}\right)=\left[\xi\left(t_{0}\right), \infty\right)_{\mathbb{T}}$ in $\left(C_{4}\right)$, we see that the derivative ${ }^{\Delta}$ on $\mathbb{T}$ is equal to the derivative $\widetilde{\Delta}$ on $\widetilde{\mathbb{T}}:=\xi\left(\left[t_{0}, \infty\right)_{\mathbb{T}}\right)$ in (34). Thus, from (34) we have

$$
(x \circ \xi)^{\Delta}=\left(x^{\Delta} \circ \xi\right) \xi^{\Delta} .
$$

Hence, from (33) and (35) we obtain

$$
w^{\Delta} \leq-Q a+a_{+}^{\Delta} \frac{w^{\sigma}}{a^{\sigma}}-a \frac{w^{\sigma}}{a^{\sigma}} \frac{\left(x^{\Delta} \circ \xi\right) \xi^{\Delta}}{x \circ \xi} .
$$

It follows from (30) that $x^{\Delta} \circ \xi \geq z^{\Delta} \circ \xi>0$. Thus, from (36) we find

$$
w^{\Delta} \leq-Q a+a_{+}^{\Delta} \frac{w^{\sigma}}{a^{\sigma}}-a \frac{w^{\sigma}}{a^{\sigma}} \frac{\left(z^{\Delta} \circ \xi\right) \xi^{\Delta}}{x \circ \xi} .
$$

Since $r(t) z^{\Delta}(t)$ is strictly decreasing on $\left[t_{2}, \infty\right)_{\mathbb{T}}$ and $\xi(t) \leq t \leq \sigma(t)$, we get $(r \circ \xi)\left(z^{\Delta} \circ\right.$ $\xi) \geq\left(r z^{\Delta}\right)^{\sigma}$ and $z^{\Delta} \circ \xi \geq\left(r z^{\Delta}\right)^{\sigma} /(r \circ \xi)>0$. Therefore, from (37) and then from (31) we conclude

$$
\begin{aligned}
w^{\Delta} & \leq-Q a+a_{+}^{\Delta} \frac{w^{\sigma}}{a^{\sigma}}-a \frac{w^{\sigma}}{a^{\sigma}} \frac{\left(r z^{\Delta}\right)^{\sigma}}{(r \circ \xi)} \frac{\xi^{\Delta}}{(x \circ \xi)} \\
& =-Q a+a_{+}^{\Delta} \frac{w^{\sigma}}{a^{\sigma}}-\frac{a \xi^{\Delta}}{r \circ \xi}\left(\frac{w^{\sigma}}{a^{\sigma}}\right)^{2} \frac{(x \circ \xi)^{\sigma}}{x \circ \xi} .
\end{aligned}
$$

From $\left(C_{4}\right)$ we see that $\xi(t)$ is strictly increasing on $\left[t_{0}, \infty\right)_{\mathbb{T}}$. Since $t \leq \sigma(t)$, we have $\xi(t) \leq \xi^{\sigma}(t)$. In view of (30), we obtain $\left(x \circ \xi^{\sigma}\right)(t) \geq(x \circ \xi)(t)>0$. Hence, from (38) there 
exists a sufficiently large $t_{5} \in\left[t_{4}, \infty\right)_{\mathbb{T}}$ such that

$$
\begin{aligned}
w^{\Delta} & \leq-Q a+a_{+}^{\Delta} \frac{w^{\sigma}}{a^{\sigma}}-\frac{a \xi^{\Delta}}{r \circ \xi}\left(\frac{w^{\sigma}}{a^{\sigma}}\right)^{2} \\
& =-Q a+\frac{\left(a_{+}^{\Delta}\right)^{2}(r \circ \xi)}{4 a \xi^{\Delta}}-\left[\frac{a_{+}^{\Delta}}{2} \sqrt{\frac{r \circ \xi}{a \xi^{\Delta}}}-\sqrt{\frac{a \xi^{\Delta}}{(r \circ \xi)}} \frac{w^{\sigma}}{a^{\sigma}}\right]^{2} \\
& \leq-Q a+\frac{\left(a_{+}^{\Delta}\right)^{2}(r \circ \xi)}{4 a \xi^{\Delta}} \text { on }\left[t_{5}, \infty\right)_{\mathbb{T}} .
\end{aligned}
$$

Integrating both sides of the last inequality from $t_{5}$ to $t$, we obtain

$$
w(t)-w\left(t_{5}\right) \leq-\int_{t_{5}}^{t}\left[a(s) Q(s)-\frac{\left(a_{+}^{\Delta}(s)\right)^{2} r(\xi(s))}{4 a(s) \xi^{\Delta}(s)}\right] \Delta s \quad \text { for } t \in\left[t_{5}, \infty\right)_{\mathbb{T}} .
$$

Since $w(t)>0$ for $t \in\left[t_{4}, \infty\right)_{\mathbb{T}}$, we have

$$
\int_{t_{5}}^{t}\left[a(s) Q(s)-\frac{\left(a_{+}^{\Delta}(s)\right)^{2} r(\xi(s))}{4 a(s) \xi^{\Delta}(s)}\right] \Delta s \leq w\left(t_{5}\right)-w(t)<w\left(t_{5}\right) \quad \text { for } t \in\left[t_{5}, \infty\right)_{\mathbb{T}} .
$$

Therefore, we conclude

$$
\limsup _{t \rightarrow \infty} \int_{t_{5}}^{t}\left[a(s) Q(s)-\frac{\left(a_{+}^{\Delta}(s)\right)^{2} r(\xi(s))}{4 a(s) \xi^{\Delta}(s)}\right] \Delta s \leq w\left(t_{5}\right)<\infty,
$$

which contradicts (18). Thus, the proof is complete.

Theorem 3.2 Assume that $\left(\mathrm{C}_{1}\right)-\left(\mathrm{C}_{6}\right)$ hold. Furthermore, suppose that there exists a positive function $a \in C_{\mathrm{rd}}^{1}\left(\left[t_{0}, \infty\right)_{\mathbb{T}}, \mathbb{R}\right)$ such that for every sufficiently large $T$,

$$
\limsup _{t \rightarrow \infty} \int_{T}^{t}\left\{a(s)\left[L_{1} p(s)-L_{2} q(v(s)) v^{\Delta}(s)\right]-\varepsilon a_{+}^{\Delta}(s)\right\} \Delta s=\infty,
$$

where $\varepsilon>0$ is an arbitrary constant and $a_{+}^{\Delta}(s):=\max \left\{a^{\Delta}(s), 0\right\}$. Then all the solutions of (1) are oscillatory.

Proof Assume that $x$ is a nonoscillatory solution of (1). Without loss of generality, we may assume that $x$ is an eventually positive solution of (1). Proceeding as in the proof of Theorem 3.1, we obtain (39). It follows from (39) that

$$
w^{\Delta} \leq-Q a+\varepsilon a_{+}^{\Delta} \text { on }\left[t_{5}, \infty\right)_{\mathbb{T}},
$$

where $\varepsilon:=w^{\sigma} / a^{\sigma}>0$. Integrating both sides of (41) from $t_{5}$ to $t$, we have

$$
w(t)-w\left(t_{5}\right) \leq-\int_{t_{5}}^{t}\left[a(s) Q(s)-\varepsilon a_{+}^{\Delta}(s)\right] \Delta s \quad \text { for } t \in\left[t_{5}, \infty\right)_{\mathbb{T}} .
$$

In view of the fact that $w(t)>0$ for $t \in\left[t_{4}, \infty\right)_{\mathbb{T}}$, we get

$$
\int_{t_{5}}^{t}\left[a(s) Q(s)-\varepsilon a_{+}^{\Delta}(s)\right] \Delta s \leq w\left(t_{5}\right)-w(t)<w\left(t_{5}\right) \quad \text { for } t \in\left[t_{5}, \infty\right)_{\mathbb{T}} .
$$


Thus, we find $\lim \sup _{t \rightarrow \infty} \int_{t_{5}}^{t}\left[a(s) Q(s)-\varepsilon a_{+}^{\Delta}(s)\right] \Delta s \leq w\left(t_{5}\right)<\infty$, which contradicts (40). Hence, the proof is complete.

Theorem 3.3 Assume that $\left(\mathrm{C}_{1}\right)-\left(\mathrm{C}_{6}\right)$ hold and that there exist a positive function $a \in$ $C_{\mathrm{rd}}^{1}\left(\left[t_{0}, \infty\right)_{\mathbb{T}}, \mathbb{R}\right)$ and functions $G, g \in C_{\mathrm{rd}}(\mathbb{D}, \mathbb{R})$, where $\mathbb{D}:=\left\{(t, s) \in \mathbb{T} \times \mathbb{T}: t \geq s \geq t_{0}\right\}$, such that

$$
G(t, t)=0 \quad \text { for } t \geq t_{0} \text { and } \quad G(t, s)>0 \text { for }(t, s) \in \mathbb{D}_{0} \text {, }
$$

where $\mathbb{D}_{0}:=\left\{(t, s) \in \mathbb{T} \times \mathbb{T}: t>s \geq t_{0}\right\}$. Furthermore, suppose that $G$ has an $r d$-continuous delta partial derivative $G^{\Delta_{s}}(t, s)$ on $\mathbb{D}$ with respect to the second variable and satisfies

$$
G^{\Delta_{s}}(t, s)+G(t, s) \frac{a_{+}^{\Delta}(s)}{a^{\sigma}(s)}=\frac{g(t, s)}{a^{\sigma}(s)} \sqrt{G(t, s)} \quad \text { for }(t, s) \in \mathbb{D}
$$

and

$$
\begin{gathered}
\limsup _{t \rightarrow \infty} \frac{1}{G(t, T)} \int_{T}^{t}\left\{G(t, s) a(s)\left[L_{1} p(s)-L_{2} q(v(s)) v^{\Delta}(s)\right]\right. \\
\left.-\frac{\left[g_{+}(t, s)\right]^{2} r(\xi(s))}{4 a(s) \xi^{\Delta}(s)}\right\} \Delta s=\infty
\end{gathered}
$$

for every sufficiently large $T$, where $a_{+}^{\Delta}(s):=\max \left\{a^{\Delta}(s), 0\right\}$ and $g_{+}(t, s):=\max \{g(t, s), 0\}$. Then all the solutions of (1) are oscillatory.

Proof Assume that $x$ is a nonoscillatory solution of (1). Without loss of generality, assume that $x$ is an eventually positive solution of (1). Proceeding as in the proof of Theorem 3.1, we have (39). Multiplying (39) by $G(t, s)$ and then integrating from $t_{5}$ to $t$, we obtain

$$
\begin{aligned}
\int_{t_{5}}^{t} G(t, s) Q(s) a(s) \Delta s \leq & -\int_{t_{5}}^{t} G(t, s) w^{\Delta}(s) \Delta s+\int_{t_{5}}^{t} G(t, s) \frac{a_{+}^{\Delta}(s)}{a^{\sigma}(s)} w^{\sigma}(s) \Delta s \\
& -\int_{t_{5}}^{t} G(t, s) \Psi(s)\left[w^{\sigma}(s)\right]^{2} \Delta s,
\end{aligned}
$$

where $\Psi(s):=\frac{a(s) \xi^{\Delta}(s)}{r(\xi(s))\left[a^{\sigma}(s)\right]^{2}}$. Making use of the formula (8), we conclude

$$
\begin{aligned}
-\int_{t_{5}}^{t} G(t, s) w^{\Delta}(s) \Delta s & =[-G(t, s) w(s)]_{s=t_{5}}^{s=t}+\int_{t_{5}}^{t} G^{\Delta_{s}}(t, s) w^{\sigma}(s) \Delta s \\
& =G\left(t, t_{5}\right) w\left(t_{5}\right)+\int_{t_{5}}^{t} G^{\Delta_{s}}(t, s) w^{\sigma}(s) \Delta s .
\end{aligned}
$$

From (45) and (46), we have

$$
\begin{aligned}
& \int_{t_{5}}^{t} G(t, s) a(s) Q(s) \Delta s \\
& \quad \leq G\left(t, t_{5}\right) w\left(t_{5}\right) \\
& \quad+\int_{t_{5}}^{t}\left\{\left[G^{\Delta_{s}}(t, s)+G(t, s) \frac{a_{+}^{\Delta}(s)}{a^{\sigma}(s)}\right] w^{\sigma}(s)-G(t, s) \Psi(s)\left[w^{\sigma}(s)\right]^{2}\right\} \Delta s .
\end{aligned}
$$


Using (43) in (47), we get

$$
\begin{aligned}
& \int_{t_{5}}^{t} G(t, s) a(s) Q(s) \Delta s \\
& \quad \leq G\left(t, t_{5}\right) w\left(t_{5}\right)+\int_{t_{5}}^{t}\left\{\frac{g(t, s)}{a^{\sigma}(s)} \sqrt{G(t, s)} w^{\sigma}(s)-G(t, s) \Psi(s)\left[w^{\sigma}(s)\right]^{2}\right\} \Delta s \\
& \quad \leq G\left(t, t_{5}\right) w\left(t_{5}\right)+\int_{t_{5}}^{t}\left\{\frac{g_{+}(t, s)}{a^{\sigma}(s)} \sqrt{G(t, s)} w^{\sigma}(s)-G(t, s) \Psi(s)\left[w^{\sigma}(s)\right]^{2}\right\} \Delta s \\
& \quad=G\left(t, t_{5}\right) w\left(t_{5}\right)+\int_{t_{5}}^{t}\left\{\frac{\left[g_{+}(t, s)\right]^{2}}{4\left[a^{\sigma}(s)\right]^{2} \Psi(s)}-\left[\frac{g_{+}(t, s)}{2 a^{\sigma}(s) \sqrt{\Psi(s)}}-\sqrt{G(t, s) \Psi(s)} w^{\sigma}(s)\right]^{2}\right\} \Delta s \\
& \quad \leq G\left(t, t_{5}\right) w\left(t_{5}\right)+\int_{t_{5}}^{t} \frac{\left[g_{+}(t, s)\right]^{2}}{4\left[a^{\sigma}(s)\right]^{2} \Psi(s)} \Delta s \\
& \quad=G\left(t, t_{5}\right) w\left(t_{5}\right)+\int_{t_{5}}^{t} \frac{\left[g_{+}(t, s)\right]^{2} r(\xi(s))}{4 a(s) \xi^{\Delta}(s)} \Delta s,
\end{aligned}
$$

where $g_{+}(t, s)$ is defined as in Theorem 3.3. Hence, it follows from (48) that

$$
\frac{1}{G\left(t, t_{5}\right)} \int_{t_{5}}^{t}\left\{G(t, s) a(s) Q(s)-\frac{\left[g_{+}(t, s)\right]^{2} r(\xi(s))}{4 a(s) \xi^{\Delta}(s)}\right\} \Delta s \leq w\left(t_{5}\right)
$$

and

$$
\limsup _{t \rightarrow \infty} \frac{1}{G\left(t, t_{5}\right)} \int_{t_{5}}^{t}\left\{G(t, s) a(s) Q(s)-\frac{\left[g_{+}(t, s)\right]^{2} r(\xi(s))}{4 a(s) \xi^{\Delta}(s)}\right\} \Delta s \leq w\left(t_{5}\right)<\infty,
$$

which contradicts (44). Therefore, this completes the proof.

Remark 3.1 The results in this paper are of higher degree of generality. From Theorems 3.1-3.3, we can obtain many different sufficient conditions for the oscillation of (1) with different choices of the functions $a, G$ and $g$. For instance, let $a(s)=1$, then we derive the following result from Theorem 3.1 or Theorem 3.2.

Corollary 3.1 Assume that $\left(\mathrm{C}_{1}\right)-\left(\mathrm{C}_{6}\right)$ and the following condition hold:

$$
\int_{T}^{\infty}\left[L_{1} p(s)-L_{2} q(v(s)) v^{\Delta}(s)\right] \Delta s=\infty
$$

for every sufficiently large $T$. Then all the solutions of (1) are oscillatory.

Let $a(s)=s$, then from Theorem 3.1 we have the following corollary.

Corollary 3.2 Assume that $\left(\mathrm{C}_{1}\right)-\left(\mathrm{C}_{6}\right)$ and the following condition hold:

$$
\limsup _{t \rightarrow \infty} \int_{T}^{t}\left\{s\left[L_{1} p(s)-L_{2} q(v(s)) v^{\Delta}(s)\right]-\frac{r(\xi(s))}{4 s \xi^{\Delta}(s)}\right\} \Delta s=\infty
$$

for every sufficiently large $T$. Then every solution of $(1)$ is oscillatory. 
Let $G(t, s)=(t-s)^{m}$ for $(t, s) \in \mathbb{D}$, where $m \geq 1$ is a constant, then $G^{\Delta_{s}}(t, s) \leq-m(t-$ $\sigma(s))^{m-1} \leq 0$ for $(t, s) \in \mathbb{D}$ (see Remark 3.3 in [45]). Take $a(s)=1$ and let $g \in C_{\mathrm{rd}}(\mathbb{D}, \mathbb{R})$ satisfy (43), then $g(t, s) \leq 0$ and $g_{+}(t, s)=0$ for $(t, s) \in \mathbb{D}$. In this case, Theorem 3.3 implies the following result.

Corollary 3.3 Suppose that $\left(\mathrm{C}_{1}\right)-\left(\mathrm{C}_{6}\right)$ hold and that there exists a constant $m \geq 1$ such that for every sufficiently large $T$,

$$
\lim _{t \rightarrow \infty} \frac{1}{t^{m}} \int_{T}^{t}(t-s)^{m}\left[L_{1} p(s)-L_{2} q(v(s)) v^{\Delta}(s)\right] \Delta s=\infty .
$$

Then all the solutions of (1) are oscillatory.

\section{Examples}

Example 4.1 Consider the second-order nonlinear dynamic equation

$$
\begin{aligned}
& {\left[t x^{\Delta}(t)\right]^{\Delta}+\frac{1}{t} x\left(t-c k_{1}\right)\left[3+x^{2}\left(t-c k_{1}\right)\right]} \\
& -\frac{1}{t \sigma(t)} \frac{2 x\left(t-c k_{2}\right)}{1+x^{2}\left(t-c k_{2}\right)}=0 \quad \text { for } t \in\left[t_{0}, \infty\right)_{\mathbb{T}},
\end{aligned}
$$

where $c>0$ is a constant, $k_{1}, k_{2}$ are positive integers, $k_{1}>k_{2}, \mathbb{T}=c \mathbb{Z}:=\{c k: k \in \mathbb{Z}\}, \sigma$ is the forward jump operator on $\mathbb{T}$, and $t_{0} \in \mathbb{T}$ satisfies $t_{0}>c\left(k_{1}-k_{2}\right)$ and $3\left[t-c\left(k_{1}-k_{2}\right)\right]^{2}+$ $3 c\left[t-c\left(k_{1}-k_{2}\right)\right]-2 t>0$ for $t \in\left[t_{0}, \infty\right)_{\mathbb{T}}$.

In (52), $r(t)=t, p(t)=\frac{1}{t}, q(t)=\frac{1}{t \sigma(t)}=\frac{1}{t(t+c)}, \xi(t)=t-c k_{1}, \delta(t)=t-c k_{2}, f(u)=u\left(3+u^{2}\right)$ and $h(u)=2 u /\left(1+u^{2}\right)$. Hence, we have $\int_{t_{0}}^{\infty} \frac{1}{r(t)} \Delta t=\int_{t_{0}}^{\infty} \frac{1}{t} \Delta t=\infty, v(t)=t-c\left(k_{1}-k_{2}\right)<t$ for $t \in \mathbb{T}, f(u) / u>3:=L_{1}$ and $0<h(u) / u<2:=L_{2}$ for $u \neq 0,|h(u)| \leq 1:=M$ on $\mathbb{R}$,

$$
\begin{aligned}
L_{1} p(t)-L_{2} q(v(t)) v^{\Delta}(t) & =\frac{3}{t}-\frac{2}{\left[t-c\left(k_{1}-k_{2}\right)\right]\left[t-c\left(k_{1}-k_{2}\right)+c\right]} \\
& =\frac{3\left[t-c\left(k_{1}-k_{2}\right)\right]^{2}+3 c\left[t-c\left(k_{1}-k_{2}\right)\right]-2 t}{t\left[t-c\left(k_{1}-k_{2}\right)\right]\left[t-c\left(k_{1}-k_{2}\right)+c\right]} \\
& >0 \quad \text { for } t \in\left[t_{0}, \infty\right)_{\mathbb{T}}
\end{aligned}
$$

and

$$
\begin{aligned}
& \int_{t}^{\infty} {\left[\frac{1}{r(s)} \int_{v(s)}^{s} q(u) \Delta u\right] \Delta s } \\
& \quad=\int_{t}^{\infty}\left[\frac{1}{s} \int_{s-c\left(k_{1}-k_{2}\right)}^{s} \frac{1}{u \sigma(u)} \Delta u\right] \Delta s \\
& \quad=\int_{t}^{\infty}\left[\frac{1}{s} \int_{s-c\left(k_{1}-k_{2}\right)}^{s}\left(-\frac{1}{u}\right)^{\Delta} \Delta u\right] \Delta s=\int_{t}^{\infty}\left[\left.\frac{1}{s}\left(-\frac{1}{u}\right)\right|_{s-c\left(k_{1}-k_{2}\right)} ^{s}\right] \Delta s \\
& \quad=\int_{t}^{\infty}\left[\frac{1}{s}\left(-\frac{1}{s}+\frac{1}{s-c\left(k_{1}-k_{2}\right)}\right)\right] \Delta s=\int_{t}^{\infty} \frac{c\left(k_{1}-k_{2}\right)}{s^{2}\left[s-c\left(k_{1}-k_{2}\right)\right]} \Delta s \\
& \quad<\infty \text { for every } t \in\left[t_{0}, \infty\right)_{\mathbb{T}} .
\end{aligned}
$$


Thus, it is easy to see that $\left(C_{1}\right)-\left(C_{6}\right)$ hold. To apply Corollary 3.1 , it remains to satisfy the condition (49). For every sufficiently large $T$, since

$$
\begin{aligned}
& \int_{T}^{\infty} L_{1} p(s) \Delta s=\int_{T}^{\infty} \frac{3}{s} \Delta s=\infty \\
& \int_{T}^{\infty} L_{2} q(v(s)) v^{\Delta}(s) \Delta s=\int_{T}^{\infty} \frac{2}{\left[s-c\left(k_{1}-k_{2}\right)\right]\left[s-c\left(k_{1}-k_{2}\right)+c\right]} \Delta s<\infty
\end{aligned}
$$

we get $\int_{T}^{\infty}\left[L_{1} p(s)-L_{2} q(v(s)) v^{\Delta}(s)\right] \Delta s=\infty$, which implies that (49) holds. Therefore, by Corollary 3.1 every solution of (52) is oscillatory.

Example 4.2 Consider the second-order nonlinear dynamic equation

$$
\left[\frac{1}{\sqrt{t}} x^{\Delta}(t)\right]^{\Delta}+\frac{1}{t^{2}} x\left(q_{0}^{-1} t\right)\left[q_{0}^{5}\left(1+q_{0}\right)+x^{2}\left(q_{0}^{-1} t\right)\right]-\frac{t+\sigma(t)}{t^{2} \sigma^{2}(t)} \frac{q_{0} x\left(q_{0}^{2} t\right)}{1+x^{4}\left(q_{0}^{2} t\right)}=0
$$

for $t \in\left[t_{0}, \infty\right)_{\mathbb{T}}$, where $q_{0}>1$ is a constant, $\mathbb{T}=\left\{q_{0}^{k}: k \in \mathbb{Z}\right\} \cup\{0\}, \sigma$ is the forward jump operator on $\mathbb{T}, t_{0} \in \mathbb{T}$ and $t_{0}>1$.

In (53), $r(t)=\frac{1}{\sqrt{t}}, p(t)=\frac{1}{t^{2}}, q(t)=\frac{t+\sigma(t)}{t^{2} \sigma^{2}(t)}, \xi(t)=q_{0}^{-1} t, \delta(t)=q_{0}^{2} t, f(u)=u\left[q_{0}^{5}\left(1+q_{0}\right)+u^{2}\right]$ and $h(u)=q_{0} u /\left(1+u^{4}\right)$. Therefore, we obtain $\int_{t_{0}}^{\infty} \frac{1}{r(t)} \Delta t=\int_{t_{0}}^{\infty} \sqrt{t} \Delta t=\infty, v(t)=q_{0}^{-3} t<t$ for $t \in\left[t_{0}, \infty\right)_{\mathbb{T}}, f(u) / u>q_{0}^{5}\left(1+q_{0}\right):=L_{1}$ and $0<h(u) / u<q_{0}:=L_{2}$ for $u \neq 0,|h(u)| \leq$ $\left(3^{3 / 4} q_{0}\right) / 4:=M$ on $\mathbb{R}$,

$$
L_{1} p(t)-L_{2} q(v(t)) v^{\Delta}(t)=\frac{q_{0}^{5}\left(1+q_{0}\right)}{t^{2}}-q_{0} \frac{q_{0}^{-3} t+q_{0}^{-2} t}{\left(q_{0}^{-3} t\right)^{2}\left(q_{0}^{-2} t\right)^{2}} q_{0}^{-3}=q_{0}^{5}\left(1+q_{0}\right)\left(\frac{1}{t^{2}}-\frac{1}{t^{3}}\right)>0
$$

for $t \in\left[t_{0}, \infty\right)_{\mathbb{T}}$, and

$$
\begin{aligned}
\int_{t}^{\infty} & {\left[\frac{1}{r(s)} \int_{v(s)}^{s} q(u) \Delta u\right] \Delta s } \\
\quad & \int_{t}^{\infty}\left[\sqrt{s} \int_{q_{0}^{-3} s}^{s} \frac{u+\sigma(u)}{u^{2} \sigma^{2}(u)} \Delta u\right] \Delta s \\
\quad & \int_{t}^{\infty}\left[\sqrt{s} \int_{q_{0}^{-3} s}^{s}\left(-\frac{1}{u^{2}}\right)^{\Delta} \Delta u\right] \Delta s=\int_{t}^{\infty}\left[\left.\sqrt{s}\left(-\frac{1}{u^{2}}\right)\right|_{q_{0}^{-3} s} ^{s}\right] \Delta s \\
& =\int_{t}^{\infty}\left[\sqrt{s}\left(-\frac{1}{s^{2}}+\frac{1}{q_{0}^{-6} s^{2}}\right)\right] \Delta s=\int_{t}^{\infty} \frac{q_{0}^{6}-1}{s^{3 / 2}} \Delta s \\
& <\infty \quad \text { for every } t \in\left[t_{0}, \infty\right)_{\mathbb{T}} .
\end{aligned}
$$

Then one can find that $\left(\mathrm{C}_{1}\right)-\left(\mathrm{C}_{6}\right)$ hold. We will apply Corollary 3.2 and it remains to satisfy the condition (50). For every sufficiently large $T$, since

$$
\begin{aligned}
& \int_{T}^{\infty} s L_{1} p(s) \Delta s=\int_{T}^{\infty} \frac{q_{0}^{5}\left(1+q_{0}\right)}{s} \Delta s=\infty \\
& \int_{T}^{\infty} s L_{2} q(v(s)) v^{\Delta}(s) \Delta s=\int_{T}^{\infty} s \frac{q_{0}^{5}\left(1+q_{0}\right)}{s^{3}} \Delta s=\int_{T}^{\infty} \frac{q_{0}^{5}\left(1+q_{0}\right)}{s^{2}} \Delta s<\infty \\
& \int_{T}^{\infty} \frac{r(\xi(s))}{4 s \xi^{\Delta}(s)} \Delta s=\int_{T}^{\infty} \frac{1}{4 q_{0}^{-3 / 2} s^{3 / 2}} \Delta s<\infty
\end{aligned}
$$


we conclude

$$
\limsup _{t \rightarrow \infty} \int_{T}^{t}\left\{s\left[L_{1} p(s)-L_{2} q(v(s)) v^{\Delta}(s)\right]-\frac{r(\xi(s))}{4 s \xi^{\Delta}(s)}\right\} \Delta s=\infty,
$$

which yields that (50) holds. Hence, by Corollary 3.2 every solution of (53) is oscillatory.

Remark 4.1 In Example 4.2, we have

$$
\int_{t_{0}}^{\infty}\left[L_{1} p(s)-L_{2} q(v(s)) v^{\Delta}(s)\right] \Delta s=\int_{t_{0}}^{\infty} q_{0}^{5}\left(1+q_{0}\right)\left(\frac{1}{s^{2}}-\frac{1}{s^{3}}\right) \Delta s<\infty,
$$

which implies that (49) does not hold. Therefore, Corollary 3.1 cannot be applied to (53).

Example 4.3 Consider the second-order nonlinear differential equation

$$
\begin{gathered}
{\left[t^{1 / 3} x^{\prime}(t)\right]^{\prime}+\frac{1}{t} x\left(t^{1 / 3}-6\right)\left[2+\sin ^{2} x\left(t^{1 / 3}-6\right)\right]} \\
-\frac{15}{t^{31}} \frac{4 x\left(t^{5}-6\right)}{1+x^{2}\left(t^{5}-6\right)}=0 \quad \text { for } t \in[2, \infty) .
\end{gathered}
$$

In (54), $\mathbb{T}=\mathbb{R}, t_{0}=2, r(t)=t^{1 / 3}, p(t)=\frac{1}{t}, q(t)=\frac{15}{t^{31}}, \xi(t)=t^{1 / 3}-6, \delta(t)=t^{5}-6, f(u)=$ $u\left(2+\sin ^{2} u\right)$ and $h(u)=4 u /\left(1+u^{2}\right)$. Thus, we get $\int_{t_{0}}^{\infty} \frac{1}{r(t)} d t=\int_{t_{0}}^{\infty} t^{-1 / 3} d t=\infty, v(t)=t^{1 / 15}<t$ for $t \in[2, \infty), f(u) / u \geq 2:=L_{1}$ and $0<h(u) / u<4:=L_{2}$ for $u \neq 0,|h(u)| \leq 2:=M$ on $\mathbb{R}$,

$$
L_{1} p(t)-L_{2} q(v(t)) v^{\Delta}(t)=\frac{2}{t}-\frac{4}{t^{3}}=\frac{2\left(t^{2}-2\right)}{t^{3}}>0 \text { for } t \in[2, \infty)
$$

and

$$
\begin{aligned}
\int_{t}^{\infty}\left[\frac{1}{r(s)} \int_{v(s)}^{s} q(u) d u\right] d s & =\int_{t}^{\infty}\left(s^{-1 / 3} \int_{s^{1 / 15}}^{s} \frac{15}{u^{31}} d u\right) d s \\
& =\int_{t}^{\infty} \frac{1}{2}\left(\frac{1}{s^{7 / 3}}-\frac{1}{s^{91 / 3}}\right) d s<\infty \quad \text { for every } t \in[2, \infty)
\end{aligned}
$$

Hence, we see that $\left(C_{1}\right)-\left(C_{6}\right)$ hold. In order to apply Corollary 3.3 , it remains to satisfy the condition (51). Take $m=1$. For every sufficiently large $T$, since

$$
\begin{aligned}
\lim _{t \rightarrow \infty} & \frac{1}{t^{m}} \int_{T}^{t}(t-s)^{m}\left[L_{1} p(s)-L_{2} q(v(s)) v^{\Delta}(s)\right] d s \\
\quad & =\lim _{t \rightarrow \infty} \frac{1}{t} \int_{T}^{t}(t-s)\left(\frac{2}{s}-\frac{4}{s^{3}}\right) d s \\
\quad & \lim _{t \rightarrow \infty}\left[2(\ln t-\ln T)+2\left(\frac{1}{t^{2}}-\frac{1}{T^{2}}\right)-\frac{2(t-T)}{t}-\frac{4}{t}\left(\frac{1}{t}-\frac{1}{T}\right)\right]=\infty,
\end{aligned}
$$

we find that (51) holds. Thus, by Corollary 3.3 every solution of (54) is oscillatory. 


\section{Authors' contributions}

The first author discovered the topic and the main ideas for the proof of the paper and made the actual writing. All authors discussed the paper together. The second and the third authors discovered some helpful ideas for the proof of this paper and checked the proof of the paper. All authors read and approved the final manuscript.

\section{Author details}

${ }^{1}$ College of Science, Hunan Institute of Engineering, 88 East Fuxing Road, Xiangtan, Hunan 411104, P.R. China. ${ }^{2}$ School of Information \& Engineering, Henan Institute of Science and Technology, Xinxiang, Henan 453003, P.R. China. ${ }^{3}$ School of Information Engineering, Xiangtan University, Xiangtan, Hunan 411105, P.R. China.

\section{Acknowledgements}

The authors would like to express their deep gratitude to the anonymous referees for their valuable suggestions and comments, which helped the authors to improve the previous manuscript of the article. This work was supported by the National Natural Science Foundation of P.R. China (Grants No. 11271311 and No. 61104072) and the Natural Science Foundation of Hunan of P.R. China (Grant No. 11JJ3010).

\section{Received: 18 January 2013 Accepted: 27 May 2013 Published: 13 June 2013}

\section{References}

1. Bohner, M, Peterson, A: Dynamic Equations on Time Scales: An Introduction with Applications. Birkhäuser, Boston (2001)

2. Bohner, M, Peterson, A: Advances in Dynamic Equations on Time Scales. Birkhäuser, Boston (2003)

3. Hilger, S: Analysis on measure chains - a unified approach to continuous and discrete calculus. Results Math. 18 18-56 (1990). doi:10.1007/BF03323153

4. Došlý, O, Hilger, S: A necessary and sufficient condition for oscillation of the Sturm-Liouville dynamic equation on time scales. J. Comput. Appl. Math. 141, 147-158 (2002). doi:10.1016/S0377-0427(01)00442-3

5. Medico, AD, Kong, Q: Kamenev-type and interval oscillation criteria for second-order linear differential equations on a measure chain. J. Math. Anal. Appl. 294, 621-643 (2004). doi:10.1016/j.jmaa.2004.02.040

6. Medico, AD, Kong, Q: New Kamenev-type oscillation criteria for second-order differential equations on a measure chain. Comput. Math. Appl. 50, 1211-1230 (2005). doi:10.1016/j.camwa.2005.07.002

7. Sun, YG: New Kamenev-type oscillation criteria of second-order nonlinear differential equations with damping. J. Math. Anal. Appl. 291, 341-351 (2004). doi:10.1016/j.jmaa.2003.11.008

8. Saker, SH: Oscillation of nonlinear dynamic equations on time scales. Appl. Math. Comput. 148, 81-91 (2004). doi:10.1016/S0096-3003(02)00829-9

9. Erbe, L, Peterson, A: Boundedness and oscillation for nonlinear dynamic equations on a time scale. Proc. Am. Math. Soc. 132, 735-744 (2004). doi:10.1090/S0002-9939-03-07061-8

10. Zhang, BG, Zhu, S: Oscillation of second-order nonlinear delay dynamic equations on time scales. Comput. Math Appl. 49, 599-609 (2005). doi:10.1016/j.camwa.2004.04.038

11. Şahiner, Y: Oscillation of second-order delay differential equations on time scales. Nonlinear Anal. 63, 1073-1080 (2005). doi:10.1016/j.na.2005.01.062

12. Erbe, L, Peterson, A, Saker, SH: Oscillation criteria for second-order nonlinear delay dynamic equations. J. Math. Anal. Appl. 333, 505-522 (2007). doi:10.1016/j.jmaa.2006.10.055

13. Jia, B, Erbe, L, Peterson, A: Kiguradze-type oscillation theorems for second order superlinear dynamic equations on time scales. Can. Math. Bull. 54, 580-592 (2011). doi:10.4153/CMB-2011-034-4

14. Karpuz, B, Öcalan, Ö: Necessary and sufficient conditions on asymptotic behaviour of solutions of forced neutral delay dynamic equations. Nonlinear Anal. 71, 3063-3071 (2009). doi:10.1016/j.na.2009.01.218

15. Karpuz, B, Öcalan, Ö, Rath, RN: Necessary and sufficient conditions for the oscillatory and asymptotic behaviour of solutions to neutral delay dynamic equations. Electron. J. Differ. Equ. 2009, 64 (2009)

16. Karpuz, B, Padhy, LN, Rath, RN: Oscillation and asymptotic behaviour of a higher order neutral differential equation with positive and negative coefficients. Electron. J. Differ. Equ. 2008, 113 (2008)

17. Grace, SR, Graef, JR, El-Beltagy, MA: On the oscillation of third order neutral delay dynamic equations on time scales, Comput. Math. Appl. 63, 775-782 (2012). doi:10.1016/j.camwa.2011.11.042

18. Senel, MT: Kamenev-type oscillation criteria for the second-order nonlinear dynamic equations with damping on time scales. Abstr. Appl. Anal. 2012, Article ID 253107 (2012). doi:10.1155/2012/253107

19. Mert, R: Oscillation of higher-order neutral dynamic equations on time scales. Adv. Differ. Equ. 2012, 68 (2012). doi:10.1186/1687-1847-2012-68

20. Zhang, C, Li, T, Agarwal, RP, Bohner, M: Oscillation results for fourth-order nonlinear dynamic equations. Appl. Math Lett. 25, 2058-2065 (2012). doi:10.1016/j.aml.2012.04.018

21. Grace, SR, Graef, JR, Zafer, A: Oscillation of integro-dynamic equations on time scales. Appl. Math. Lett. 26, 383-386 (2013). doi:10.1016/j.aml.2012.10.001

22. Saker, SH, Grace, SR: Oscillation criteria for quasi-linear functional dynamic equations on time scales. Math. Slovaca 62, 501-524 (2012). doi:10.2478/s12175-012-0026-9

23. Candan, T: Oscillation of second-order nonlinear neutral dynamic equations on time scales with distributed deviating arguments. Comput. Math. Appl. 62, 4118-4125 (2011). doi:10.1016/j.camwa.2011.09.062

24. Grace, SR, Agarwal, RP, Zafer, A: Oscillation of higher order nonlinear dynamic equations on time scales. Adv. Differ. Equ. 2012, 67 (2012). doi:10.1186/1687-1847-2012-67

25. Hassan, TS: Kamenev-type oscillation criteria for second order nonlinear dynamic equations on time scales. Appl. Math. Comput. 217, 5285-5297 (2011). doi:10.1016/j.amc.2010.11.052

26. Li, T, Han, Z, Sun, S, Zhao, Y: Oscillation results for third order nonlinear delay dynamic equations on time scales. Bull. Malays. Math. Soc. 34, 639-648 (2011)

27. Zhang, Q: Oscillation of second-order half-linear delay dynamic equations with damping on time scales. J. Comput. Appl. Math. 235, 1180-1188 (2011). doi:10.1016/j.cam.2010.07.027 
28. Thandapani, E, Piramanantham, V, Pinelas, S: Oscillation criteria for second-order neutral delay dynamic equations with mixed nonlinearities. Adv. Differ. Equ. 2011, Article ID 513757 (2011). doi:10.1155/2011/513757

29. Saker, SH, O’Regan, D: New oscillation criteria for second-order neutral functional dynamic equations via the generalized Riccati substitution. Commun. Nonlinear Sci. Numer. Simul. 16, 423-434 (2011). doi:10.1016/j.cnsns.2009.11.032

30. Zhang, Q, Qiu, F: Oscillation theorems for second-order half-linear delay dynamic equations with damping on time scales. Comput. Math. Appl. 62, 4185-4193 (2011). doi:10.1016/j.camwa.2011.10.002

31. Sun, Y: Interval oscillation criteria for second-order dynamic equations with nonlinearities given by Riemann-Stieltjes integrals. Abstr. Appl. Anal. 2011, Article ID 719628 (2011). doi:10.1155/2011/719628

32. Han, Z, Li, T, Sun, S, Zhang, C, Han, B: Oscillation criteria for a class of second-order neutral delay dynamic equations of Emden-Fowler type. Abstr. Appl. Anal. 2011, Article ID 653689 (2011). doi:10.1155/2011/653689

33. Zafer, A: On oscillation and nonoscillation of second-order dynamic equations. Appl. Math. Lett. 22, 136-141 (2009). doi:10.1016/j.aml.2008.03.003

34. Karpuz, B: Unbounded oscillation of higher-order nonlinear delay dynamic equations of neutral type with oscillating coefficients. Electron. J. Qual. Theory Differ. Equ. 2009, 34 (2009)

35. $\mathrm{Xu}, \mathrm{Y}, \mathrm{Xu}, \mathrm{Z}$ : Oscillation criteria for two-dimensional dynamic systems on time scales. J. Comput. Appl. Math. 225, 9-19 (2009). doi:10.1016/j.cam.2008.06.010

36. Chen, DX: Oscillation and asymptotic behavior for $n$ th-order nonlinear neutral delay dynamic equations on time scales. Acta Appl. Math. 109, 703-719 (2010). doi:10.1007/s10440-008-9341-0

37. Braverman, E, Karpuz, B: Nonoscillation of first-order dynamic equations with several delays. Adv. Differ. Equ. 2010 Article ID 873459 (2010). doi:10.1155/2010/873459

38. Erbe, L, Hassan, TS, Peterson, A: Oscillation of third order nonlinear functional dynamic equations on time scales. Differ. Equ. Dyn. Syst. 18, 199-227 (2010). doi:10.1007/s12591-010-0005-y

39. Ünal, M, Zafer, A: Oscillation of second-order mixed-nonlinear delay dynamic equations. Adv. Differ. Equ. 2010, Article ID 389109 (2010). doi:10.1155/2010/389109

40. Lin, Q, Jia, B, Wang, Q: Forced oscillation of second-order half-linear dynamic equations on time scales. Abstr. Appl. Anal. 2010, Article ID 294194 (2010). doi:10.1155/2010/294194

41. Sun, S, Han, Z, Zhao, P, Zhang, C: Oscillation for a class of second-order Emden-Fowler delay dynamic equations on time scales. Adv. Differ. Equ. 2010, Article ID 642356 (2010). doi:10.1155/2010/642356

42. Zhang, SY, Wang, QR: Oscillation of second-order nonlinear neutral dynamic equations on time scales. Appl. Math. Comput. 216, 2837-2848 (2010). doi:10.1016/j.amc.2010.03.134

43. Sun, $T, X i, H$, Peng, X, Yu, W: Nonoscillatory solutions for higher-order neutral dynamic equations on time scales. Abstr. Appl. Anal. 2010, Article ID 428963 (2010). doi:10.1155/2010/428963

44. Karpuz, B: Asymptotic behaviour of bounded solutions of a class of higher-order neutral dynamic equations. Appl. Math. Comput. 215, 2174-2183 (2009). doi:10.1016/j.amc.2009.08.013

45. Saker, $\mathrm{SH}$ : Oscillation of second-order nonlinear neutral delay dynamic equations on time scales. J. Comput. Appl. Math. 187, 123-141 (2006). doi:10.1016/j.cam.2005.03.039

doi:10.1186/1687-1847-2013-168

Cite this article as: Chen et al.: Oscillation of second-order nonlinear dynamic equations with positive and negative coefficients. Advances in Difference Equations 2013 2013:168.

\section{Submit your manuscript to a SpringerOpen ${ }^{\circ}$ journal and benefit from:}

- Convenient online submission

- Rigorous peer review

- Immediate publication on acceptance

Open access: articles freely available online

- High visibility within the field

- Retaining the copyright to your article 